\title{
Application of Soft Computing Models to Daily Average Temperature Analysis
}

\author{
Mustafa Göçken *†, Aslı Boru*, Ayşe Tuğba Dosdoğru**, Nafiz Berber*** \\ *Adana Science and Technology University, Industrial Engineering Department, Adana, Turkey \\ **Gaziantep University, Industrial Engineering Department, Gaziantep, Turkey \\ ***Orcan Natural Gas Wholesale and Distribution Company Limited, Hatay, Turkey \\ mgocken@adanabtu.edu.tr, aboru@adanabtu.edu.tr, dosdogru@gantep.edu.tr
}

+ Corresponding author; Mustafa Gocken, Industrial Engineering Department, Adana Science and Technology University,

Yeşiloba Yerleşkesi, Yeşiloba mah., Ögretmenler Bulvarı,

46278 sok., No: 3, 01180, Seyhan/ADANA, Tel.: 0 (322) 4550000 - 2226;

Fax: 0 (322) 45500 09, Email: mgocken@adanabtu.edu.tr

Received: 02.04.2015 Accepted:12.06.2015

\begin{abstract}
Providing critical information about daily life, weather forecasting has important role for human being. Especially, temperature forecasting is rather important because it affects not only people but also other atmospheric parameters. Various techniques have been used for analysis of the dynamic behaviour of weather. This ranges from simple observation of weather to using computer technology. In this study, ANFIS (Adaptive Network Based Fuzzy Inference System), ANN (Artificial Neural Network) and MRA (Multiple Regression Analysis) have been applied for weather forecasting. To judge the forecasting capability of the proposed models, the graphical analysis and the indicators of the accuracy of Mean Absolute Deviation (MAD), Mean Square Error (MSE), Root-Mean Squared Error (RMSE), Mean Absolute Percent Error (MAPE), Determination Coefficient $\left(\mathrm{R}^{2}\right)$, Index of Agreement (IA), Fractional Variance (FV), Coefficient of Variation (CV, \%) are given to describe models' forecasting performance and the error. The results show that ANFIS exhibited best forecasting performance on weather forecasting compared to ANN and MRA.
\end{abstract}

Keywords Weather forecasting, ANFIS, ANN, MRA.

\section{Introduction}

Weather is a description of instant conditions of the atmosphere at a particular time. Weather directly and indirectly affects our daily life because it affects the main ingredients of life on Earth such as soil, water etc. To ensure quality life, weather parameters should be analysed carefully. For these reasons, many people are applied their own model to predict these parameter. Over the many years, the state of the cloud, wind direction, intensity of the stars, and many other observational factors are used to take precautions. In this way, they save lives, money and time in both local and global area. However, this prediction doesn't provide sufficient information because weather is affected by number of variables. For this purpose, meteorological services carried out challenging operational tasks to understand weather [1] and so many types of models are prepared to solve mystery of weather. Presenting a linear relationship between input data and output data is one of the most popular one. One of the simple models to present linear relationship is Multiple Linear Regression (MLR). Paras and Mathur [2] used MLR to develop a model for weather forecasting. In the study, data collected from a particular station and then applied to estimate weather conditions. Some statistical indicators such as moving average are also processed to extract the hidden information of the time series. Abatzoglou et al. [3] presented general structure of seasonal temperature and precipitation trends by using MLR models. The result of the study shows that MLR models explained between $22 \%$ and $54 \%$ of the interannual variance in seasonal temperature. The other powerful model is ANN that is an important alternative tool to conventional models in weather forecasting. ANN has a reasonable forecasting accuracy and minimum forecasting error. The properties of ANN are well suited to the problem of weather forecasting under temporal and spatial variability [4]. Abhishek et al. [5] presented applicability of ANN and developed nonlinear predictive models for weather analysis. In the study, MSE is used as a measure of the forecasting accuracy. The results show that this study can be helpful to concentrate on the trend of 
weather over a long period of time in a particular station and area. In same manner, Dombayc1 and Gölcü [6] used ANN in daily temperature forecasting and evaluated ANN accuracy using FV and RMSE. Smith et al. [7] used ANN to forecast air temperature based on near real-time data. Smith et al. [8] developed an enhanced ANN for air temperature forecasting including larger training set sizes, seasonal input terms, increased lag lengths, and varying the size of the network. Şahin [9] modelled monthly mean air temperature based on remote sensing and ANN. Hayati, and Mohebi [10] utilized ANN for one day ahead forecasting of an important weather parameter and used MAE to evaluate ANN model. Kaur and Singh [11] used ANN to forecast the minimum temperature for Chandigarh city. Previous studies presented that weather forecasting with ANN are very encouraging and forecasting can be made with high degree of accuracy. In addition to ANN, combining ANN and fuzzy logic called ANFIS is used in many application of the weather forecasting. Quality of a weather forecasting is increased by fuzziness that characterizes the scattering of real data around the prognosis [12]. Also, the human thinking and reasoning are easily described using fuzzy logic in the mathematical framework. Thus, fuzzy logic is principle of many systems and ANFIS is one of them. ANFIS reasonably forecast a large class of functions and it presents dynamic behaviour of atmospheric conditions in a clear way. Tektaş [13] used ANFIS and Auto Regressive Moving Average models for weather forecasting in Turkey. The performance comparisons of ANFIS and ARIMA models due to MAE, RMSE, $\mathrm{R}^{2}$ presented that ANFIS yields better results. Daneshmand et al. [14] used ANFIS for modelling and forecasting the monthly minimum temperature. Research results indicated that the ANFIS can forecast the monthly minimum temperature in the particular station. Oyediran and Adeyemo [15] used ANFIS and MultiLayer Perceptron to represent characteristic of metrological data sets. It is found that ANFIS model has an ability to yield better results than the Multi-Layer Perceptron model. Also, ANFIS clearly analysed more compact and natural internal representation of the temperature, rainfall, wind, and relative humidity. It is seen that researchers continue to develop finer models for increasing accuracy in forecasting. In this study, ANFIS, ANN, and MRA have been applied for weather forecasting.

\section{Preprocessing}

Selection of input variables is a fundamental task. The task of selecting input variables is largely dependent on the discovery of relationships within the available data to identify suitable predictors of the model output. Using too much unnecessary input data may overload the system, reducing the calculation speed and at times worsen the results of the forecasting system [16]. In literature, many researchers have applied trial and error according to their experiences to determine input variables. Similarly, we used trial and error method. On the other hand, researchers should be careful in this step. Defining what constitutes an optimal set of input variables is significant in designing forecasting models. Identification of an optimal set of input variables will lead to a more accurate, efficient, cost-effective, and more easily interpretable models [16]. We are interested in quantifying the strength and direction of a presumed relationship between input variables and temperature. In these cases, we can use a measure of association known as a correlation to determine the characteristics of this relationship. This complex relationship between input variables and temperature is shown in Fig. 1-4. For this purposes, we used the Pearson product-moment correlation coefficient that is the most common statistical calculation. Usually known as the "Pearson r'" or simply " $r$,', this statistic is a measure of the covariance of the two variables divided by the product of their standard deviation. The higher the absolute value of the correlation coefficient, the stronger the correlation between the two weather parameters. Note that a positive correlation indicates an increasing linear relationship, while a negative correlation indicates a decreasing linear relationship [17]. It may take on a range of values from -1 to 0 to +1 , where the values are absolute and nondimensional with no units involved. The strength of the correlation is not dependent on the direction or the sign. Thus, $r=0.40$ and $r=-0.40$ are equal in the degree of association of the measured variable [18]. For a correlation coefficient, we are interested in how two variables vary with respect to each other. There are two important assumptions of the Pearson correlation coefficient: first, it can be used only with interval or ratio data and second, the data must be normally distributed. Also, it should be noted that no matter how strong the correlation coefficient, we cannot say that one variable cause the other.

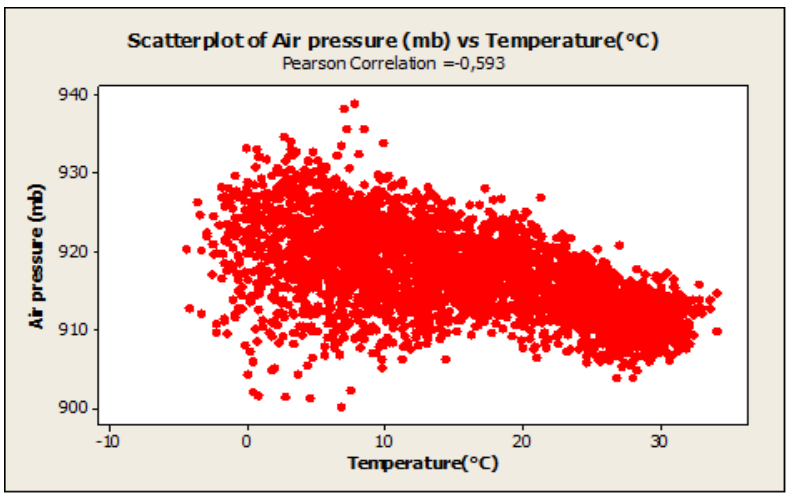

Fig. 1. The scatter plot of air pressure-temperature.

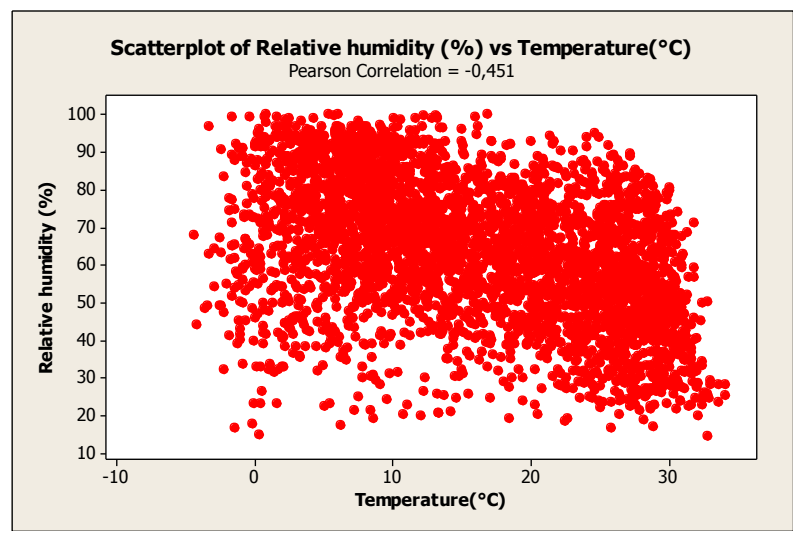

Fig. 2. The scatter plot of relative humidity-temperature. 


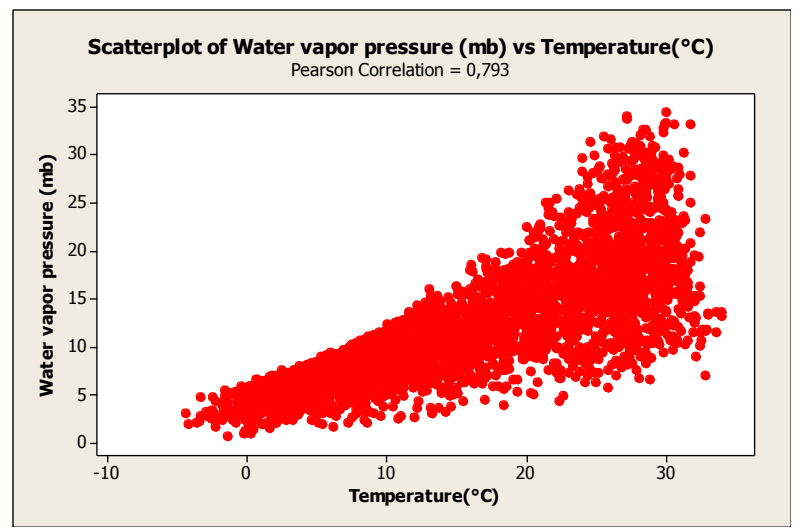

Fig. 3. The scatter plot of water vapour pressure-temperature.

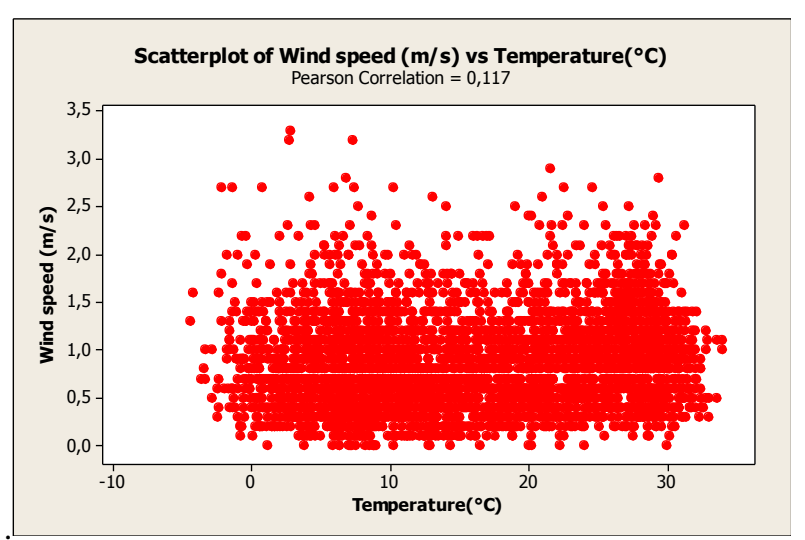

Fig. 4. The scatter plot of wind speed-temperature.

We can only report the strength and direction of the relationship [19]. The scatter plot is useful to illustrate the concept of correlation and to visualize the relationship between the input variables. In this study, scatter plots are generated for the correlations $-0.593,-0.451,0.793$, and 0.117 that are the Pearson product-moment correlation coefficient. That is, the higher the correlation in either positive direction or negative direction, the more linear the association between two variables and the more obvious the trend in a scatter plot. The scatter plot of water vapour pressure and temperature shows approximately linear trend but others are not clear as water vapour pressure and temperature. It is also seen that temperature and air pressure are negatively correlated. This means that as the temperature decreases, the air pressure increases, and as the temperature increases, the air pressure decreases. In same manner, humidity is negatively correlated with temperature. The wind speed is slightly correlated with temperature. Data are daily average values for Gaziantep between 01/01/2001 and $31 / 12 / 2010$ and obtained from General Directorate of Meteorology. The number of data which are 18260 is sufficient to apply ANFIS, ANN, and MRA for weather forecasting. Details about data are given in Table 1.
Table 1. Descriptive Statistics of the input variables and output variable

\begin{tabular}{|c|c|c|c|c|c|}
\hline & $\mathrm{N}$ & Mean & $\begin{array}{c}\text { Std. } \\
\text { Deviation }\end{array}$ & Minimum & Maximum \\
\hline Air pressure & 3652 & 916,7691 & 5,51781 & 900,10 & 938,70 \\
\hline $\begin{array}{c}\text { Water vapor } \\
\text { pressure }\end{array}$ & 3652 & 12,1347 & 6,31326 & 0,70 & 34,30 \\
\hline Humidity & 3652 & 63,7321 & 18,28262 & 14,30 & 100,00 \\
\hline Wind speed & 3652 & 0,8661 & 0,48689 & 0,00 & 3,30 \\
\hline Temperature & 3652 & 15,9233 & 9,29021 & $-4,40$ & 34,00 \\
\hline
\end{tabular}

\section{ANFIS}

ANFIS has a potential to capture the benefits of ANN and fuzzy logic in a single model. Linguistic information is utilized from the fuzzy logic. The learning capability and parameter optimization is taken from ANN. Also, ANN learning rules are applied to identify and set the parameters and structure of a Fuzzy Inference System (FIS). Basically, ANFIS design includes two steps. First step is the design of the premise parameters and the other step is consequent parameter training. ANFIS keeps the premise parameters fixed and estimates them in a forward pass and then in a backward pass by keeping fixed the consequent parameters [20]. ANFIS has adaptable structure and includes developed data analysing technique such as numerical classification and constructing a rule. Hence, all rules formed for weather forecasting can be appointed by ANFIS [21]. The basic structure of FIS defines the number and type of the membership functions used in the fuzzy rules, and a reasoning mechanism of FIS. Note that FIS does not have a learning algorithm for parameter estimation but it is able to employ rules and knowledge that are represented with linguistic expressions [22]. Let $\mathrm{U}$, a subset of $\mathrm{R}$, be the universe discourse, where $U=\left\{u_{1}, u_{2}, \ldots, u_{n}\right\}$, in which the possible linguistic values $f_{A(T)}$ of fuzzy sets $\mathrm{A}(\mathrm{t})$ are defined, where $f_{A(T)}$ denotes the membership function of the fuzzy set $\mathrm{A}(\mathrm{t}), f_{A(T)}: U \rightarrow[0,1]$, and the $\mathrm{A}(\mathrm{t})$ can be seen as a linguistic variable which is a collection of $\mathrm{f}_{\mathrm{A}(\mathrm{T})}\left(\mathrm{u}_{1}\right), \mathrm{f}_{\mathrm{A}(\mathrm{T})}\left(\mathrm{u}_{2}\right), \ldots, \mathrm{f}_{\mathrm{A}(\mathrm{T})}\left(\mathrm{u}_{\mathrm{n}}\right) . \quad \mathrm{F}=\{\mathrm{A}(\mathrm{t}), \mathrm{t}=1,2, \ldots\}$ is called a fuzzy time series defined on $U$. Assume the temperature on the first day is 39 , denoted as Temperature $(1)=39$, while the temperature on the second day is 34 , denoted as Temperature $(2)=34$, and the temperature on day $\mathrm{t}$ is 39.5 , denoted as Temperature $(t)=39.5$ (Fig. 5). Following the fuzzification process, each of these temperature values would then obtain a linguistic value; for example, $\mathrm{A}(1)=$ "Very high temperature", $\mathrm{A}(2)=$ "High temperature", $\mathrm{A}(\mathrm{t}-1)=$ "High temperature", $\mathrm{A}(\mathrm{t})=$ "Very high temperature". 

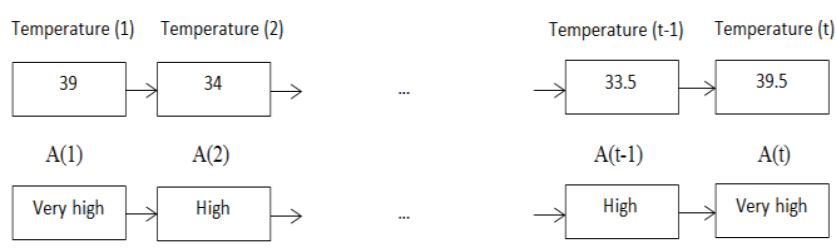

Fig. 5. Illustrative example of linguistic expressions.

Thus, the $\mathrm{F}$ is a fuzzy time series definition on $\mathrm{U}$, and $\mathrm{F}=$ \{"Very high", "High",..., "High", "Very high"\} [23]. Inputoutput mapping smoothly constructed by using ANFIS serves as a basis for building the set of fuzzy if-then rules to generate the input output pairs [21]. Based on FIS, ANFIS uses the fuzzy rule base as the model structure to transform the original rules into fuzzy rules one by one. Hence, by the fuzzy clustering of data or other clustering analysis, huge amounts of data can be transformed into fuzzy rule bases, thus reducing parameter computation and data storage requirements. In addition, expert experience and knowledge can be transformed into inference rules to compensate for the lack of a data-based description of the system [24]. Therefore, the main contribution of ANFIS in the modeling process is the fuzzy if-then rules, in which the membership function parameters are estimated with the learning algorithm of ANN. Using the learning capability of ANN, the fuzzy rules are automatically generated and the parameters are optimized [22]. To give two fuzzy if-then rules example, the two rules is given for a first order Sugeno model as follow [25]:

Rule 1: If $\mathrm{v}$ is $\mathrm{V}_{1}$ and $\mathrm{d}$ is $\mathrm{D}_{1}$ then $f_{1}=p_{1} v+q_{1} d+r_{1}$

Rule 1: If $\mathrm{v}$ is $\mathrm{V}_{2}$ and $\mathrm{d}$ is $\mathrm{D}_{2}$ then $f_{2}=p_{2} v+q_{2} d+r_{2}$

In Eq. (1) and Eq. (2), $p_{1}, p_{2}, q_{1}, q_{2}, r_{1}$ and $r_{2}$ are linear parameters and $V_{1}, V_{2}, D_{1}$ and $D_{2}$ are non-linear parameters. A circle indicates a fixed node whereas a square indicates an adaptive node i.e. the parameter are changed during adaptation or training and $O_{j, i}$, denotes the output of the $\mathrm{i}^{\text {th }}$ node in layer $\mathrm{j}$. The entire system structure consists of five layers (Fig. 6).

\section{Layer 1}

Each node ' $i$ ' in this layer generates a membership grades of a linguistic label. It is the fuzzy layer, in which $\mathrm{v}$ and $d$ are the input of nodes. $V_{1}, V_{2}, D_{1}$, and $D_{2}$ are linguistic labels used in the fuzzy theory for dividing the membership functions. The membership relationship between the output and input functions of this layer can be expressed as given in Eq. (3) and Eq. (4):

$$
\begin{array}{ll}
O_{l, i}=\mu_{V i}(\mathrm{v}) & \mathrm{i}=1,2 \\
O_{l, j}=\mu_{D j}(\mathrm{~d}) & \mathrm{j}=1,2
\end{array}
$$

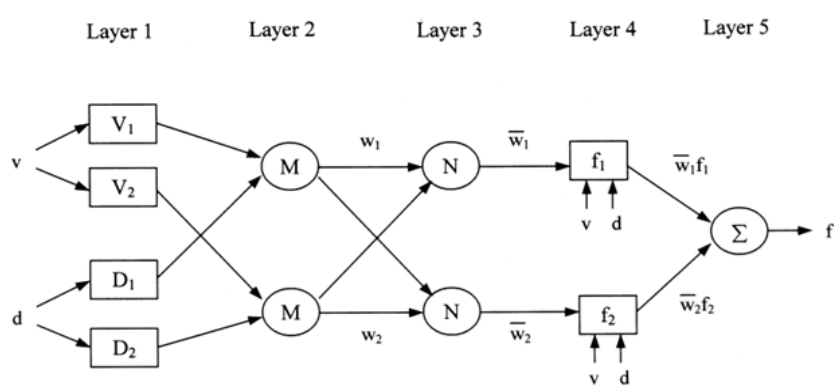

Fig. 6. Basic ANFIS structure.

where $O_{l, i}$ and $O_{l, j}$ denote the output functions and $\mu_{V i}(\mathrm{v})$, $\mu_{D j}$ (d) denote the membership functions. The membership function which represents a fuzzy set is usually denoted by $\mu_{V i}(\mathrm{v})$ and $\mu_{D j}(\mathrm{~d})$. For an element $\mathrm{d}$ and $\mathrm{v}$, the value $\mu_{V i}(\mathrm{v})$ and $\mu_{D j}(\mathrm{~d})$ is called the membership degree of $\mathrm{d}$ and $\mathrm{v}$ in the fuzzy set. The membership degree $\mu_{V i}(\mathrm{v})$ and $\mu_{D j}$ (d) quantifies the grade of membership of the element $d$ and $v$ to the fuzzy set. If the value is $0, d$, and $v$ are not a member of the fuzzy set. If the value is $1, \mathrm{~d}$, and $\mathrm{v}$ are member of the fuzzy set. Lastly, if value is between 0 and 1 , fuzzy members only partially belong to the fuzzy set [26]. In this study, each input parameters are divided into seven scale on the membership function; very low, low, little low, medium, little high, high and very high. Thus, the triangular membership function is employed; $\mu_{V i}(v)$ is given in Eq. (5):

$$
\mu_{V i}(\mathrm{v})=\max \left[\min \left(\frac{v-a_{i}}{b_{i}-a_{i}}, \frac{c_{i}-v}{c_{i}-b_{i}}\right)\right], 0
$$

where $a_{i}, b_{i}$, and $c_{i}$ are the parameters of the membership function, governing the triangular membership functions accordingly. Triangular shape membership function is one of the most suitable membership functions to forecast temperature because it requires less computing time.

\section{Layer 2}

Each node in this layer calculates the 'firing strength' of each rule via multiplication (Eq. (6)):

$\mathrm{O}_{2, \mathrm{i}}=\mathrm{w}_{\mathrm{i}}=\mu_{\mathrm{Vi}}(\mathrm{v}) \mu_{\mathrm{Di}}(\mathrm{v}) \quad \mathrm{i}=1,2, \ldots$

where $O_{2, i}$ denotes the output of the layer 2 .

\section{Layer 3}

The $\mathrm{i}^{\text {th }}$ node of this layer calculates the ratio of the $\mathrm{i}^{\text {th }}$ rule's strength to the sum of all rules' firing strengths (Eq. (7)):

$$
\mathrm{O}_{3, \mathrm{i}}=\overline{\mathrm{w}}_{\mathrm{i}}=\frac{\mathrm{w}_{\mathrm{i}}}{\left(\mathrm{w}_{1}+\mathrm{w}_{2}\right)} \quad \mathrm{i}=1,2, \ldots
$$

For convenience, output of this layer $O_{3, i}$ will be called 'normalized firing' strength. 


\section{Layer 4}

Every node ' $i$ ' in this layer is a square node with a node function as given in Eq. (8).

$O_{4, i}=\bar{w}_{i} f_{i}=\bar{w}_{i}\left(p_{i} v+q_{i} d+r_{i}\right) \mathrm{i}=1,2, \ldots$

where $O_{4, i}$ denotes the layer 4 output. In this layer, $p_{i}, q_{i}$, and $r_{i}$ are called linear parameter or consequent parameters.

\section{Layer 5}

The single node in this layer is a fixed node, which computes the overall output as the summation of all incoming signals (Eq. (9)):

$O_{5, i}=\sum_{i} \bar{w}_{l} f_{i}=\frac{\sum_{i} w_{i} f_{i}}{\sum_{i} w_{i}} \quad \quad \mathrm{i}=1,2, \ldots$

In ANFIS structure, first and fourth layers are adaptive layers. The modifiable parameters are so-called premise parameters in the first layer and consequent parameters in the fourth layer [25].

In this paper, MATLAB is used to create ANFIS and it contains all the ANFIS information such as ANFIS editor, membership function editor, rule editor and so on. To create fuzzy inference system, either Mamdani or Sugeno type systems can be used but ANFIS has only support Sugeno type system. For this reason, Sugeno type system is used intentionally in the study. The main difficulty of the ANFIS model is related to the number of input variables. If ANFIS inputs exceed five, the computational time and rule numbers will increase, so ANFIS will not be able to model output with respect to inputs [27]. Therefore, we used four input variables. Most literature on forecasting show that triangular shape membership function is one of the most suitable membership functions to forecast temperature because it requires less computing time. Hence, we used triangular membership function and each input parameters are divided into seven scale on the membership function; very low, low, little low, medium, little high, high and very high. In ANFIS, $2401\left(7^{4}\right)$ rules are created for 4 inputs and 7 membership function defined for each input. A simple interpretation of one rule is "If air pressure is the first membership function associated with air pressure and if vapour pressure is the first membership function associated with vapour pressure, and if humidity is the first membership function associated with humidity, and if wind speed is the first membership function associated with wind speed, then output 1 should be the membership function associated with output 1 with weight 1 .

\section{ANN}

ANN is a computational network and simulates the networks of neurons of biological central nervous system [28]. One of the most important features of ANN is that it performs a large number of numerical operations in parallel. These operations involve simple arithmetic operations as well as nonlinear mappings and computation of derivatives. Almost all data stored in the network are involved in recall computation at any given time [29]. ANN has an ability to learn nonlinear problem with training that provide sufficiently accurate online response and to model unknown data relationship. Simple model of ANN has three set of rules: multiplication, summation, and activation. Artificial neuron is the basic building block of every ANN and affect system performance directly. The inputs are weighted in the model and every input value is multiplied with individual weight. Sum function (sums all weighted inputs and bias) is applied in the middle section of artificial neuron. Finally, weighted inputs and bias is passing through activation function that is also called transfer function [30]. Basic working principle of ANN is seen in Fig. 7. Also, simplicity of artificial neuron model is given in Eq. (10).

$\mathrm{y}_{\mathrm{i}}(\mathrm{k})=\mathrm{F} \cdot\left(\sum_{\mathrm{i}=0}^{\mathrm{m}}\left(w_{i}(\mathrm{k}) \cdot x_{i}(\mathrm{k})+\mathrm{b}\right)\right.$

Where:

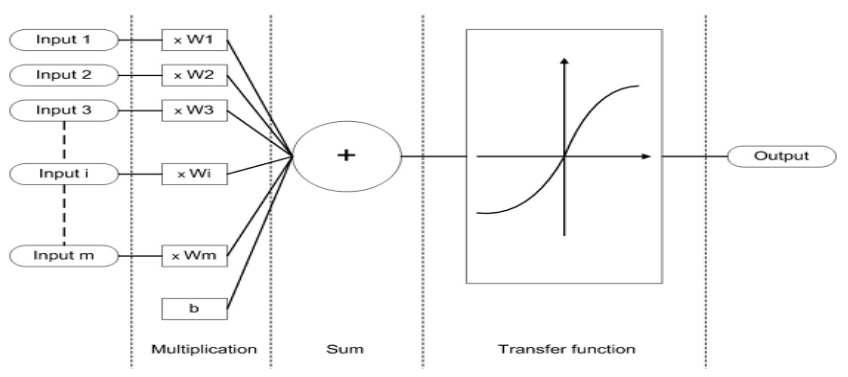

Fig. 7. Working principle of an artificial neuron [30].

$>x_{i}(\mathrm{k}), w_{i}(\mathrm{k})$, and $\mathrm{y}_{\mathrm{i}}(\mathrm{k})$ represent input value, weight value, and output value in discrete time $\mathrm{k}$ where $\mathrm{i}$ goes from 0 to $\mathrm{m}$ respectively,

$>$ b is bias,

$>\mathrm{F}$ is a transfer function.

Each connection of input layer has an independent weight, $w$ attached to it. The input parameters are fed through the input nodes to the next level where each of the input values are multiplied by the relevant weight on each connection to create a weighted input value [30]. In ANN, the input parameters that are air pressure $(\mathrm{mb})$, water vapour pressure $(\mathrm{mb})$, relative humidity $(\%)$, and wind speed $(\mathrm{m} / \mathrm{s})$ are fed through the input nodes to the next level where each of the input values are multiplied by the relevant weight on each connection to create a weighted input value. Then, system continues by determining the number of hidden layers and nodes. Although there have been some suggestions related to these decisions, a standard model does not exist. Generally these crucial decisions are determined by 
trial and error method. One hidden layer is generally enough for most applications.

As a remark, notice that a larger number of hidden neurons in general would yield better learning capability of the ANN until the overfit phenomenon occurs [31]. Choi [31] presented that 20 hidden neurons can be used in ANN because the value of the MSE is lowest when compared with other number of the hidden neurons $(2,10,15,25,30,100)$. Therefore, we used one hidden layer and 20 hidden neurons in ANN structure. In ANN, $15 \%$ of the original data are divided randomly to use as validation and test data sets. $70 \%$ of the original data are used for training. The process of training the network is the adjustment of the weights to produce the desired response to the given inputs. In ANN, an input-output pair is selected from the training set and the selected inputs are used to calculate output. Then, error that is the difference between the network output and the desired output is found. This training cycle is repeated until the error reduces to an acceptable value [6].

\section{MRA}

MRA is one of the simple statistical tools that are used for weather forecasting. One or more variables can be used to forecast another variable of interest. In the model, the input variables are known as independent variables and the variable that is being forecasted is known as the dependent variable [32]. However, the connections between dependent and independent variables are generally not known clearly and hence detail information about solution is not provided. The choice of independent variables is made by the researcher in the experimental design stage. In this study, air pressure $(\mathrm{mb})$, water vapour pressure $(\mathrm{mb})$, relative humidity $(\%)$, and wind speed $(\mathrm{m} / \mathrm{s})$ are selected as the independent variables and temperature $\left({ }^{\circ} \mathrm{C}\right)$ is selected as the dependent variable. The general form of a regression model for $\mathrm{k}$ independent variables is given in Eq. (11).

$\mathrm{Y}=\beta_{0+} \beta_{1} \mathrm{X}_{1+} \beta_{2} \mathrm{X}_{2+\ldots \ldots \ldots+} \beta_{\mathrm{k}} \mathrm{X}_{\mathrm{k}+} \epsilon$

Where $\mathrm{Y}$ is the response variable. $\beta_{0}, \beta_{1}$ to $\beta_{\mathrm{k}}$ are the regression coefficients. $\epsilon$ is the error, and $X_{1}, X_{2}, \ldots, X_{k}$ are the independent variables. Based on least squares criterion, the regression coefficients are forecasted by minimizing the sum of the squares of the vertical deviations of each data point to the best-fitting line [33].

\section{Results and Discussion}

We first present scatter plots that are probably the simplest verification tool to provide useful insights in Fig. 810. The analyses of scatter plot in proposed models are useful to get a general vision of how well the forecasting model is. The values should lie linearly in a perfect forecasting model. The ordinate and the abscissa should have the same scale, in which case perfection is represented by any point on the 45 degree line for which forecast=observed. If the forecasts were perfect, this line would coincide with the 45 degree line [34]. It could be said that forecasting performance of the proposed models are remarkable in all models. However, ANFIS and ANN results are more accurate than those of MRA's. In this study, we also used performance indicators to analyse in greater detail. An important aspect of the error indicators used for model evaluations is their capability to discriminate among model results [35]. In the literature, various descriptive statistical indicators are used as helpful tools to describe model's forecasting performance and the error. MAD is the mean of the absolute values of the error between actual and forecast data. The smallest MAD gives the most reliable results when several forecasting models are compared. Hence, the smaller the MAD values the better the forecasting performance. The MAPE is a relative measure which expresses errors as a percentage of the actual data. This is its biggest advantage as it provides as easy and intuitive way of judging the extent, or importance of errors. In addition we can make comparisons involving more than one model since the MAPE of each tells us about the average relative size of their errors [36]. MSE, as its name implies, provides for a quadratic loss function as it squares and subsequently averages the various errors. MSE is especially useful when we are concerned about large errors whose negative consequences are proportionately much bigger than equivalent smaller ones [37]. Besides MSE, RMSE is also useful when large errors are particularly undesirable [38]. $\mathrm{R}^{2}$ estimates the combined dispersion against the single dispersion of the observed and forecasted series. The range of $\mathrm{R}^{2}$ lies between 0 and 1 which describes how much of the observed dispersion is explained by the forecasting [39]. IA reflects the degree to which the observed variable is accurately forecasted by the input variables. IA is not a measure of correlation or association in the formal sense but rather a measure of the degree to which a model's forecasting are error free. It varies between 0 and 1 where a computed value of 1 indicates perfect agreement between the observation and forecasting, and 0 connotes one of a variety of complete disagreements [40]. FV is a normalization of the mean bias of the sample variances of the observed and forecasted values. $\mathrm{CV}$ is also useful to appraise the fit between the observed data and the forecasted outputs. The results of all performance indicators are summarized in Table 2. The results revealed that the forecasting performance of the ANFIS model is better than that of both ANN and MRA models according to all performance indicators. However, the difference between ANFIS and ANN is less than the difference between ANFIS and MRA. The value of the MRA steeply increases which implies that forecasting accuracy dramatically decreases. These indicate that the non-linear ANFIS and ANN models generate a better fit than MRA. One of the most important advantages of ANFIS is that ANFIS integrates ANN and fuzzy logic in a single framework. Thus, it has a potential to capture the benefits of both models. Also, ANFIS reaches to the target faster than ANN. When a more sophisticated system with a huge data is imagined, the use of ANFIS instead of ANN would be more useful to overcome faster the complexity of the problem [41]. 
Table 2. Descriptive statistical performance indicators for proposed models

\begin{tabular}{|c|c|c|c|}
\hline & ANFIS & ANN & MRA \\
\hline MAD & 1,092 & 1,31 & 2,442 \\
\hline MSE & 2,158 & 2,824 & 9,960 \\
\hline RMSE & 1,469 & 1,680 & 3,155 \\
\hline MAPE & 0,072 & 0,124 & 0,238 \\
\hline $\mathbf{R}^{2}$ & 0,975 & 0,966 & 0,885 \\
\hline IA & 0,994 & 0,991 & 0,968 \\
\hline FV & 0,013 & 0,021 & 0,059 \\
\hline (CV, \% $)$ & 9,224 & 10,551 & 19,817 \\
\hline
\end{tabular}

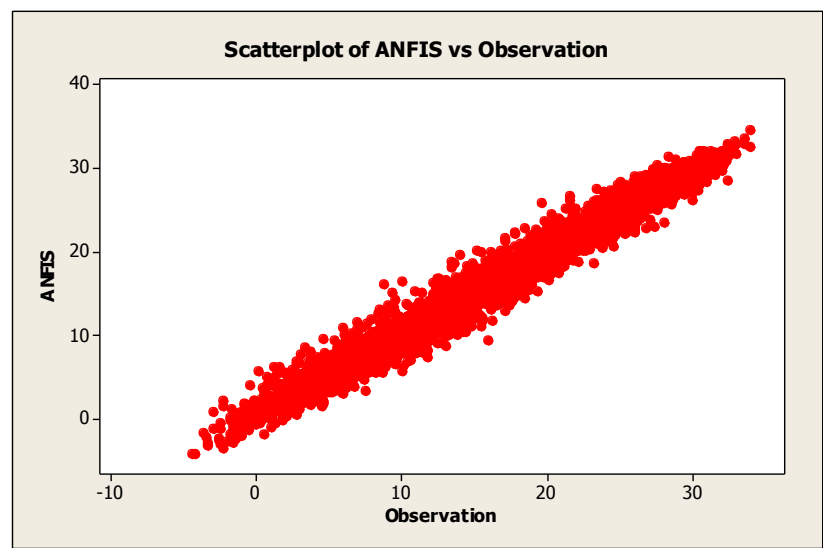

Fig. 8. Comparison of observation and forecasting value in 2010 (ANFIS).

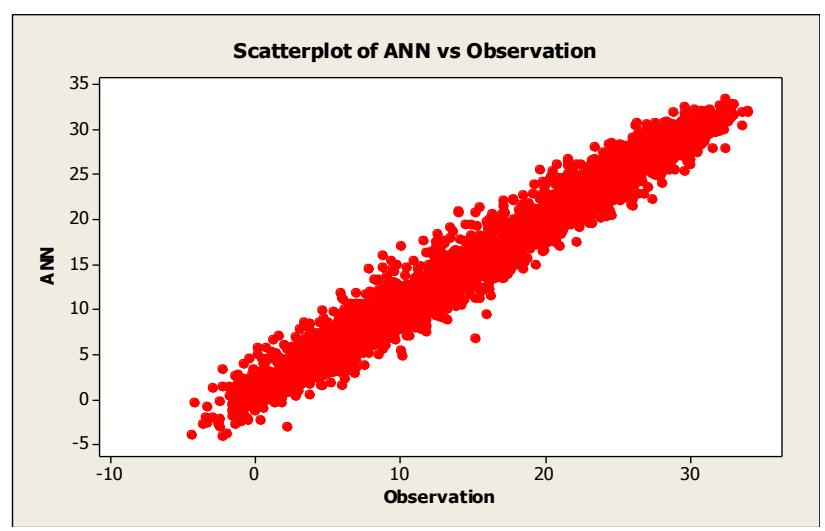

Fig. 9. Comparison of observation and forecasting value in 2010 (ANN).

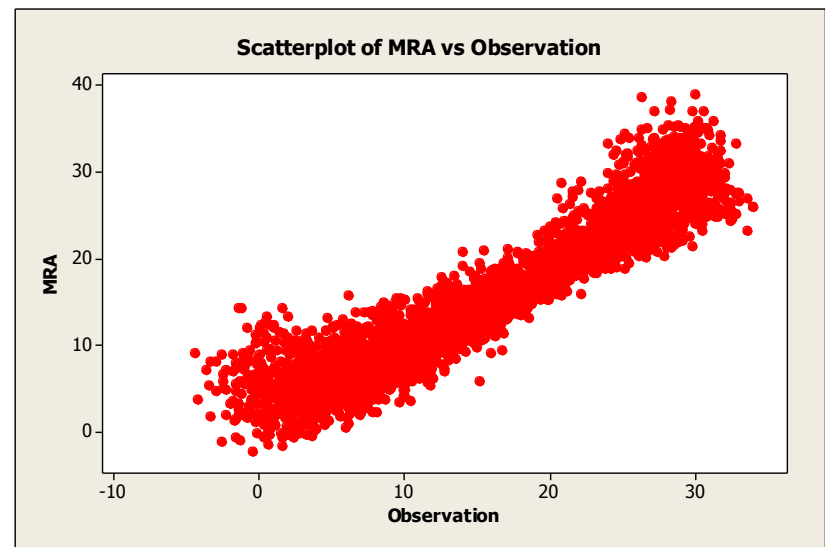

Fig. 10. Comparison of observation and forecasting value in 2010 (MRA).

\section{Conclusion}

In this study, 18260 data are used for forecasting daily average temperature of city of Gaziantep by using ANFIS, ANN, and MRA. Every forecasting model is analysed separately and results are compared. Enabling easily configurable forecasting model with given input stands out as a remarkable feature of fuzzy logic. In this study, fuzzy logic is supported by ANFIS Sugeno type system. In FIS structure, 2401 rules are used and triangular membership function is chosen for being more suitable. The other powerful method is ANN that enables to analyse the relationship between the inputs and outputs in forecasting. However, there are a number of different answers to the question of how to define ANN structure. Hence, the determination of the appropriate architecture such as the number of variables, number of layers, and number of neurons in each layer is critical in the design of ANN. We used one hidden layer and 20 hidden neurons in ANN structure. Also, MRA is used for weather forecasting but results are not satisfying as others. Also, the graphical analysis (scatter plot) and statistical performance indicators (MAD, MSE, RMSE, MAPE, $\mathrm{R}^{2}$, IA, FV, CV, \%) are utilized to judge the forecasting capability of the developed methodology. Specifically, the MAPE performances of each model tells us that the forecasting error of the proposed methods are under approximately $0,238 \%$ at the worst case which is acceptable for such a very complex forecasting environment. Also, this limit shows the superiority of the forecasting performances of the proposed methods. Based on the results of the performance indicators, it can conclude that ANFIS produced very small deviations and exhibited superior forecasting performance on weather forecasting compared to both ANN and MRA. The results emphasized that ANFIS can be used conveniently for further weather forecasting studies.

\section{References}

[1] G. Shrivastava, S. Karmakar, M. K. Kowar, and P. Guhathakurta, Application of Artificial Neural Networks in weather forecasting: A comprehensive literature review, 
International Journal of Computer Applications, vol. 51, no. 18, pp. 0975-8887, 2012.

[2] Paras, and S. Mathur, A simple weather forecasting model using mathematical regression, Indian Research Journal of Extension Education Special Issue 1, pp. 161$168,2012$.

[3] J. T. Abatzoglou, D. E. Rupp, and P. W. Mote, Seasonal climate variability and change in the Pacific Northwest of the United States, American Meteorological Society, vol. 27, pp. 2125-2142, 2014.

[4] I. Maqsood, M. R. Khan, and A. Abraham, Weather forecasting models using ensembles of neural networks, Intelligent Systems Design and Applications Advances in Soft Computing, vol. 23, pp. 33-42, 2003.

[5] K. Abhishek, M. P. Singh, S. Ghosh, and A. Anand, Weather forecasting model using Artificial Neural Network, Procedia Technology, vol. 4, pp. 311-318, 2012.

[6] Ö. A. Dombayc1, and M. Gölcü, Daily means ambient temperature prediction using Artificial Neural Network method: A case study of Turkey, Renewable Energy, vol. 34, pp. 1158-1161, 2009.

[7] B. A. Smith, G. Hoogenboom, and R. W. McClendon, Artificial Neural Networks for automated year-round temperature prediction, Computers and Electronics in Agriculture, vol. 68, pp. 52-61, 2009.

[8] B. A. Smith, R. W. McClendon, and G. Hoogenboom, An enhanced Artificial Neural Network for air temperature prediction, World Academy of Science, Engineering and Technology, vol.1 no.7, pp. 80-85, 2005.

[9] M. Şahin, Modeling of air temperature using remote sensing and Artificial Neural Network in Turkey, Advances in Space Research, vol. 50, no. 7, pp. 973-985, 2012.

[10] M. Hayati, and Z. Mohebi, Application of Artificial Neural Networks for temperature forecasting, World Academy of Science, Engineering and Technology, vol.1, no.4, pp. 654-658, 2007.

[11] A. Kaur, and H. Singh, Artificial Neural Networks in forecasting minimum temperature, International Journal of Electronics \& Communication Technology, vol. 2, no. 3, pp. 101-105, 2011.

[12] D. Domanska, and M. Wojtylak, Fuzzy weather forecast in forecasting pollution concentrations, Proc. of Chaotic Modeling and Simulation International Conference, pp. 1-8, 2010.
[13] M. Tektaş, Weather forecasting using ANFIS and ARIMA models, Environmental Research, Engineering and Management, vol. 51, pp. 5-10, 2010.

[14] H. Daneshmand, T. Tavousi, M. Khosravi, and S. Tavakoli, Modeling minimum temperature using Adaptive Neuro-Fuzzy Inference System based on spectral analysis of climate indices: A case study in Iran, Journal of the Saudi Society of Agricultural Sciences, vol. 14, pp. 33-40, 2015.

[15] O. F. Oyediran, and A. B. Adeyemo, Performance evaluation of Neural Network MLP and ANFIS models for weather forecasting studies, African Journal of Computing \& ICT, vol. 6, no. 1, pp. 147-164, 2013.

[16] R. May, G. Dandy, and H. Maier, Review of input variable selection methods for Artificial Neural Networks, Artificial Neural Networks-Methodological Advances and Biomedical Applications, pp. 19-44, 2011.

[17] N. Sharma, P. Sharma, D. Irwin, and P. Shenoy, Predicting Solar Generation from Weather Forecasts Using Machine Learning, Distributed Generation, Microgrids, Renewables and Storage (IEEE SmartGridComm), pp. 528533, 2011.

[18] R. Taylor, Interpretation of the correlation coefficient: A basic review, Journal of Diagnostic Medical Sonography, vol. 6 , pp. 35-39, 1990.

[19] S. Prion, and K. A. Haerling, Making sense of methods and measurement: pearson product-moment correlation coefficient. Clinical Simulation in Nursing, vol. 10, pp. 587$588,2014$.

[20] Dr G. S. V. P. Raju, V. M. Sumalatha, K. V. Ramani, and K. V. Lakshmi, Solving uncertain problems using ANFIS, International Journal of Computer Applications, vol. 29, no. 11, 2011.

[21] R. Sivakumar, C. Sahana, and P. A. Savitha, Design of ANFIS based estimation and control for MIMO systems, International Journal of Engineering Research and Applications, vol. 2, no. 3, pp. 2803-2809, 2012.

[22] C. Jeong, J. Shin, T. Kim, and J. Heo, Monthly precipitation forecasting with a Neuro-Fuzzy model, Water Resources Management, vol. 26, pp. 4467-4483, 2012.

[23] M. Chen, and B. Chen, Online fuzzy time series analysis based on entropy discretization and a Fast Fourier Transform, Applied Soft Computing, vol.14, pp. 156-166, 2014.

[24] M. Y. Chen, D. R. Chen, M. H. Fan, and T. Y. Huang, International transmission of stock market movements: an Adaptive Neuro-Fuzzy Inference System for analysis of 
TAIEX forecasting, Neural Computing and Applications, vol. 23, pp. 369-378, 2013.

[25] S. Roy, Design of Adaptive Neuro-Fuzzy Inference System for predicting surface roughness in turning operation, Journal of Scientific and Industrial Research, vol. 64, pp. 653-659, 2005.

[26] S. Mandal, J. Choudhury, and S. Chaudhuri, In search of suitable fuzzy membership function in prediction of time series data, International Journal of Computer Science Issues, vol. 9, no 3, pp. 293-302, 2012.

[27] B. Khoshnevisan, S. Rafiee, M. Omid, and H. Mousazadeh, Development of an intelligent system based on ANFIS for predicting wheat grain yield on the basis of energy inputs, Information Processing in Agriculture, vol. 1, pp. 14-22, 2014.

[28] D. Graupe, Principles of Artificial Neural Networks, Advanced Series on Circuits and Systems, 2st ed., vol. 6, New Jersey: World Scientific, 2007.

[29] J. M. Zurada, Introduction to Artificial Neural Systems, St. Paul: West Publishing Company, vol. 8, 1992.

[30] A. Krenker, J. Bester, and A. Kos, Introduction to the Artificial Neural Networks. Artificial Neural Networks: Methodological Advances and Biomedical Applications, First ed., Intech, India, pp 1-18, 2011.

[31] T. M. Choi, Fashion Branding and Consumer Behaviors: Scientific Models, Imprint: Springer, 2014.

[32] D. Şchiopu, E. G. Petre, and C. NegoiŃă, Weather forecast using SPSS statistical methods, Petroleum-Gas University of Ploiesti Bulletin, Mathematics-InformaticsPhysics, vol. 61, no. 1, pp. 97-100, 2009.

[33] Z. Z. Latt, and H. Wittenberg, Improving flood forecasting in a developing country: a comparative study of Stepwise Multiple Linear Regression and Artificial Neural Network, Water Resource Management, vol. 28, pp. 2109 2128, 2014.

[34] H. R. Stanski, W. R. Burrows, and L. J. Wilson, Survey of common verification methods in meteorology, (Second Edition), World Meteorological Organization, 1989.

[35] T. Chai, and R. Draxler, Root mean square error (RMSE) or mean absolute error (MAE)? - Arguments against avoiding RMSE in the literature, Geoscientific Model Development, vol. 7, pp. 1247-1250, 2014.

[36] S. Makrıdakıs, and M. Hibon, Evaluating accuracy (or error) measures, INSEAD, 1995.
[37] E. Woschnagg, Evaluating forecast accuracy, University of Vienna, Department of Economics, 2004.

[38] A. Bhatt, D. Pant, and R. Singh, An analysis of the performance of Artificial Neural Network technique for apple classification, AI \& Soc. vol. 29, 103-111, 2014.

[39] P. Krause, D. P. Boyle, and F. Bäse, Comparison of different efficiency criteria for hydrological model assessment, Advances in Geosciences, vol.5, pp. 89-97, 2005 .

[40] C. J. Willmott, On the validation of models. Physical Geography, vol. 2, pp. 194-194, 1981.

[41] H. Atmaca, B. Cetisli, and H. S. Yavuz, The comparison of fuzzy inference systems and neural network approaches with ANFIS method for fuel consumption data, Second International Conference on Electrical and Electronics Engineering Papers ELECO, 2001. 